\title{
Multiple Salmonella enteritidis leg abscesses in a patient with systemic lupus erythematosus
}

\author{
Arie Shamiss, Michael Thaler, Naomi Nussinovitch, Rika Zissin ${ }^{1}$ and Talma \\ Rosenthal
}

Departments of Medicine D and ${ }^{1}$ Roentgenology, Chaim Sheba Medical Center, Tel Hashomer 52621, and Tel Aviv University Sackler Faculty of Medicine, Israel

\begin{abstract}
Summary: We describe a 19 year old woman with systemic lupus erythematosus on corticosteroid therapy, who developed bilateral, multiple, gas-forming Salmonella enteritidis leg abscesses and osteomyelitis mimicking deep vein thrombosis. The infection was treated successfully by a combination of surgical drainage and intravenous ceftriaxone, followed by prolonged oral pefloxacin.

This rare case of gas-producing $S$. enteriditis emphasizes the difficulty in diagnosing such complications in active systemic lupus erythematosus.
\end{abstract}

\section{Introduction}

Systemic lupus erythematosus (SLE) is one of the diseases thought to predispose to severe salmonella infections. ${ }^{1-3}$ However, such infections rarely present as soft tissue abscesses: Cohen et al. ${ }^{4}$ found only one case with SLE out of 42 soft tissue infections in a recent review on extra-intestinal salmonella infections during the antibiotic era. The diagnosis is more difficult when the salmonella infection occurs during an exacerbation of SLE, as illustrated by the following case.

\section{Case report}

A 19 year old Arab woman with a one-year history of SLE, on corticosteroids, presented with a painful swelling in the right leg just below the knee, fever, and exacerbation of her underlying disease manifested by arthritis, massive proteinuria and high titres of anti-DNA. Symptoms had persisted for 2 weeks.

Physical examination revealed a woman with cushingoid appearance and a temperature of $38^{\circ} \mathrm{C}$. Heart, lungs and abdominal examination were normal. There was a $2+$ pitting oedema over her ankles and sacrum. Her hands, elbows and knees were swollen and tender, and there was a tender swelling in her right leg, extending proximally from below the knee to the ankle. The thigh and calf on both sides were oedematous and painful.

Urine collection showed a proteinuria of $15 \mathrm{~g} / 24$ hours, serum albumin $20 \mathrm{~g} / \mathrm{l}$, creatinine $70.9 \mu \mathrm{mol} / 1$.

Correspondence: A. Shamiss, M.D.

Accepted: 30 January 1990
Haemoglobin was $6.2 \mathrm{mmol} / \mathrm{l}$, white blood cell count $9000 / \mathrm{mm}^{3}$, ESR 78 in the first hour (Westergren method), antinuclear factor highly positive, and anti-DNA binding $40 \%$.

Initial diagnosis of deep vein thrombosis was made, and treatment begun with intravenous heparin. This therapy was discontinued 4 days later because of increased swelling of the right leg. Radiographs of the legs demonstrated bilateral soft tissue swelling with gas bubbles, multiple fluid levels on the right leg, and no bone involvement in either leg. Marked muscle atrophy was also evident (Figure 1). Ultrasound showed patent veins and fluid collection in both calves. Needle aspiration with the aid of ultrasound yielded $100 \mathrm{ml}$ of brown fluid, found by light microscopy to contain a few blood clots and leucocytes, suggesting an infected haematoma.

Computed tomography (CT) of the lower extremities revealed multiple fluid collections consistent with abscesses in both legs and thighs (Figure 2). A gas-producing bacterial infection was diagnosed, and surgical drainage performed. Cultures of blood and aspirated fluid yielded $S$. enteriditis. A bone scan showed increased uptake in the right tibia and left femur, correlating well with the periostal changes on the CT scan which were most probably secondary to the soft tissue infection. Stool culture was negative.

Treatment was begun with oral co-trimoxazole, and an attempt was made to taper the steroid dosage with the addition of azathioprine. However, pancytopenia soon developed and fever persisted, and the antimicrobial treatment was changed to ceftriaxone $2 \mathrm{~g} /$ day, which was replaced after one 


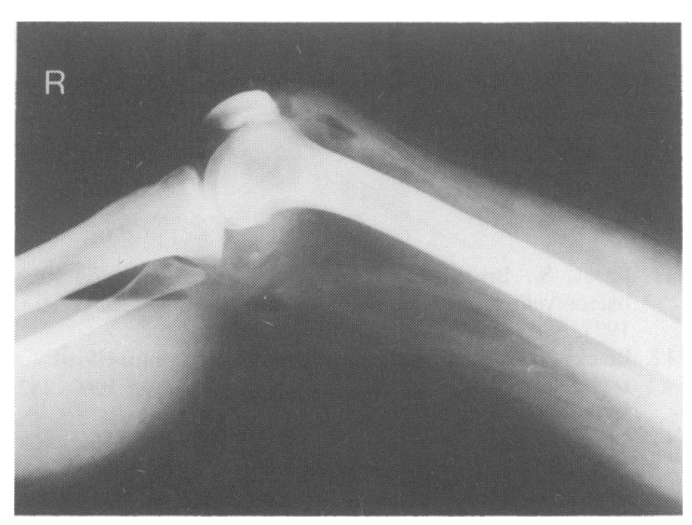

Figure 1 Radiograph of right leg with horizontal beam showing normal bones, soft tissue swelling, separation of muscles, and air-fluid levels in both the thigh and calf.

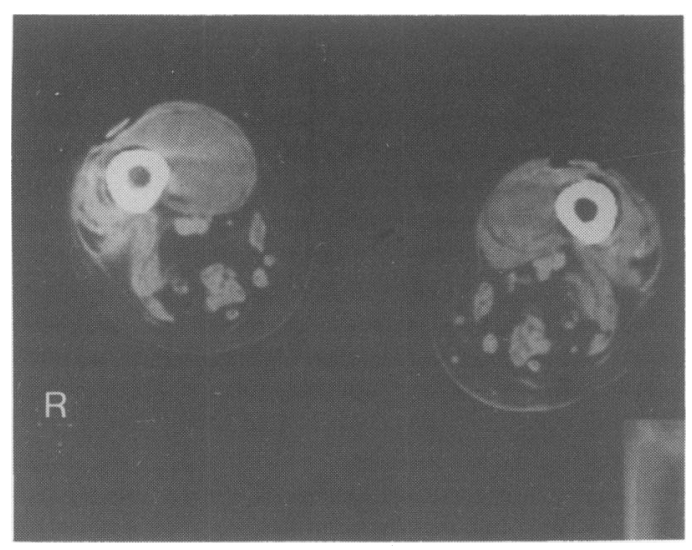

Figure 2 CT scan through the distal third of both thighs showing muscle atrophy and large bilateral fluid collections.

week by oral pefloxacin $800 \mathrm{mg} /$ day when the granulocyte count returned to normal.

Following control of the infection by surgical drainage and the described antibiotic treatment, the patient's lupus flare-up subsided: the anti-DNA reduced to $6 \%$ and the proteinuria disappeared. Computerized tomography performed one month later showed abatement of the fluid collections, and persistence of marked muscle atrophy.

The patient was discharged to an orthopaedic rehabilatation centre, where her ability to walk and function unassisted improved markedly. Since the possibility of osteomyelitis was not entirely ruled out, she continued the oral antibiotics for 3 months, according to the protocol for salmonella osteomyelitis in SLE patients described by Sattar and Molly ${ }^{5}$ and Ortiz-Neu et al. ${ }^{6}$

\section{Discussion}

Conditions predisposing to salmonella soft tissue infections and osteomyelitis are sickle cell disease, ${ }^{7}$ underlying malignancies, ${ }^{8}$ diabetes mellitus, ${ }^{9}$ renal transplants, ${ }^{10}$ previous trauma and preexisting bone disease, ${ }^{11}$ steroid therapy, ${ }^{12}$ and connective tissue disorders, including SLE. ${ }^{5,6,13,14}$

The large number of patients with underlying diseases may reflect the importance of an immune mechanism in clearing salmonella organisms. Serum from patients with sickle cell disease has been shown to have deficient complement-mediated opsonizing activity for salmonella, ${ }^{16}$ while patients with diabetes mellitus or SLE with and without corticosteroid therapy, as in our case, have a defect in their cellular immune system which may be important in salmonella infections. ${ }^{15}$ Factors in our SLE patient that might contribute to this predisposition include the use of an immunosuppressive drug (prednisone), and the impaired granulocytic and monocytic phagocytosis that has been described in SLE patients. ${ }^{3}$

The combination of salmonella soft tissue infection and osteomyelitis in a SLE patient is extremely rare, and only one such case was found by Cohen $e t$ $a{ }^{4}{ }^{4}$ in a recent review of the literature on extraintestinal salmonella infections. The two complications in these patients pose a difficult diagnostic problem, since they can be masked by the fever and joint swelling associated with an exacerbation of the underlying condition. In addition, as in our patient, the clinical presentation can be highly suggestive of acute thrombophlebitis, which sometimes occurs in SLE. Only the worsening of the calf swellings following anticoagulation therapy, and the demonstration of fluid collections on imaging, led to the correct diagnosis.

The $S$. enteriditis infection in our patient presented as bacteraemia, leg abscesses and osteomyelitis. It was demonstrated by X-ray of the patient's legs, and necessitated aggressive surgical drainage and prolonged antimicrobial therapy. To the best of our knowledge this is only the second reported case of subcutaneous salmonella-induced gas production. ${ }^{16}$

Until recently, antimicrobial therapy for systemic salmonella infection was limited to ampicillin, chloramphenicol and co-trimoxazole. ${ }^{14}$ Despite their potential role in the treatment of serious salmonella infections, there are only a few reports of successful treatment with third generation cephalosporins or quinolones. ${ }^{17-19}$

The need for long-term oral treatment in our patient led us to choose a short intravenous course of ceftriaxone followed by prolonged oral pefloxacin. Improvement expressed by normalization of temperature, sterile cultures and regression of the fluid collections was achieved over one month. The 
development of salmonella enteritidis in the form of multiple gas-forming leg abscesses and osteomyelitis signifies the kind of serious life- threatening complication that can develop in the course of a basically controlled disease.

\section{References}

1. Guerrent, R.L. Salmonella infections. In: Harrison's Principles of Internal Medicine, 11th edn. McGraw-Hill, New York, 1987, pp. 592-598.

2. Abramson, S., Kramer, S.B., Radin, A. \& Holtzman, R. Salmonella bacteremia in SLE. Arthritis Rheum 1985, 28: 75-79.

3. Lovy, M.R., Ryan, P.F.J. \& Hughes, G.R.V. Concurrent SLE and salmonellosis. J Rheumatol 1981, 8: 605-612.

4. Cohen, J.I., Bartlett, J.A. \& Corey, G.R. Extra-intestinal manifestations of Salmonella infections. Medicine 1987, 66: 349-388.

5. Satter, M.A. \& Molly, J. Salmonella osteomyelitis in a patient with SLE. J Infection 1984, 9: 93-96.

6. Ortiz-Neu, C., Marr, J.S., Cherubin, C.E. \& Neu, H.C. Bone and joint infections due to Salmonella. J Infect Dis 1978, 138: $820-828$.

7. Hook, E.W., Cambell, C.G., Weens, H.S. \& Cooper, G.R. Salmonella osteomyelitis in patients with sickle cell anemia. N Engl J Med 1957, 257: 403-407.

8. Wolfe, M.S., Armstrong, D., Louria, D.B., Blevins, A. Salmonellosis in patients with neoplastic disease. Arch Intern Med 1971, 128: 546-554.

9. Drow, D., Wegleitner, M. \& Lau, K. Neck abscess caused by Salmonella typhimurium. South Med J 1982, 75: 900.

10. Samra, Y., Shakaed, Y. \& Maier, M. Non-typhoid salmonellosis in renal transplant recipients. Rev Infect Dis 1986, 8: 431-440.

11. Porat, S., Brezis, M. \& Kopolevic, J. Salmonella typhi osteomyelitis long after a fracture. J Bone Joint Surg (Am) 1977, 59A: 687-689.

12. Black, P.H., Kunz, L.F. \& Swartz, M.N. Salmonellosis - a review of some unusual aspects. $N$ Engl $J$ Med 1960, 262: 811-817.

13. Clinico-pathological Conference 8-1968. N Engl J Med 1968, 278: $441-450$.

14. Cherubin, C.E., Neu, H.C., Imperato, P.J., Harvey, R.P. \& Bellen, N. Septicemia with nontyphoid salmonella. Medicine 1974, 53: 365-376.

15. Hand, W.L. \& King, N.L. Serum opsonization of salmonella in sickle cell anemia. Am J Med 1978, 64: 388-395.

16. Brennan, D.D. \& Grieco, M. Gas production with Salmonella typhimurium infection. Am J Med Sci 1971, 262: 255-260.

17. Brian, J.P., Rocha, H. \& Scheld, W.M. Problems in salmonellosis: rationale for clinical trials with newer beta-lactam agents and quinolones. Rev Infect Dis 1986, 8: 189-207.

18. Cherubin, C.E., Eng, R.H.K. \& Smith, S.M. Cephalosporin therapy for salmonellosis. Arch Intern Med 1986, 146: $2149-2152$.

19. Dolan, S.A., Everett, E.D. \& Harper, M.C. Salmonella vertebral osteomyelitis treated with cefotaxime. Arch Intern Med 1987, 147: 1667-1668. 